\title{
Propietarios de Facebook INC. : Principales datos estructurales y financieros
}

\author{
Owners of Facebook INC. : Main structural and financial data
}

\author{
Dra. Cristina Martín-Jiménez, Universidad de Sevilla \\ crismjpress@gmail.com| https://orcid.org/0000-0001-6518-1808 \\ Calle San Fernando, 4, 41004 Sevilla
}

\begin{abstract}
Resumen
Facebook es una nueva plataforma de Comunicación producto de la Cuarta Revolución Industrial. El mecanismo interno de la llamada red social se ha transformado en un corto espacio de tiempo, afectando las noticias e informaciones que reciben a diario millones de usuarios en todo el mundo a través de esta plataforma. Detrás de una noticia siempre están los intereses de los propietarios de los medios y canales de comunicación, ya que estos no solo son un negocio para sus accionistas privados sino que contienen una intencionalidad e interés en línea con las ambiciones de sus dueños. Los académicos críticos han presentado innumerables casos que demuestran que el Poder hace uso de las estructuras mediáticas para afianzarse y perpetuarse. El estudio del Poder conlleva analizar las múltiples categorías y elementos de los nuevos medios que surgen en cada revolución tecnológica. Y uno de los elementos que se analiza desde el enfoque de la EPICC es la estructura de propiedad.

En Facebook, su cara visible es Mark Zuckerberg, a quien suele identificarse como su propietario. Pero, ¿qué oculta la cara invisible de esta compañía global a la que se acusa de vigilar a los usuarios y de cambiar el resultado de las elecciones gubernamentales?

Aplicando el enfoque de la EPICC, vamos a analizar la estructura de propiedad de Facebook Inc. con el objetivo de conocer si los poderes de carácter financiero-mediático-político están detrás de esta plataforma.
\end{abstract}

\begin{abstract}
Facebook is a new communication platform, a result of the Fourth Industrial Revolution. Internal mechanisms of the so-called social network have changed in a short space of time, affecting news and information reported daily by millions of users across the world. Behind the news you always have the interests of media owners. Critical scholars have presented innumerable proofs showing that Power uses media structures to perpetuate its idiosyncrasies. The study of
\end{abstract}

Forma de citar:

Martin-Jiménez, C. (2019). Propietarios de Facebook INC. : Principales datos estructurales y financieros. Ámbitos. Revista Internacional de Comunicación, 44, 200-216. doi: 10.12795/Ambitos.2019.i44.12 
Power has to do with multiple categories and elements, such as new media that arise within any technological revolution. One of the analyzed elements from the EPICC approach is the structure of the property.

Facebook visible face is Mark Zuckerberg, who usually identifies himself as its owner. But, what the invisible face of this global company accused of monitoring its users and changing the outcome of government elections hide? Applying the EPICC approach, we will analyze the structure of its property.

Palabras clave: Facebook, redes sociales, Poder, propietarios de medios, estructura económica-política-mediática.

Keywords: Facebook, social networks, Power, media owners, economic-political-media structure.

\section{INTRODUCCIÓN}

Facebook Inc. se lanzó en 2004, oficialmente, como una empresa de redes sociales con servicios de noticias, mensajes y aplicaciones móviles fundada por cuatro jóvenes estudiantes de Harvard. Su semilla se plantó en la misma Universidad que el conglomerado Time Warner Inc., Allí, en sus inicios, fue una plataforma para conectar a los miembros de las fraternidades estudiantiles.

Seis años después, en 2010, Tejedor-Calvo ya subrayó que "en un tiempo reducido las redes sociales han pasado de ser un sistema dedicado a la formación de comunidades online a tener un protagonismo destacado dentro de los cibermedios, llegando incluso a asumir funciones de promoción, difusión e información" (2010: 617).

Describía, entre otros, el caso de Facebook, que en sus catorce años de vida ha pasado de considerarse una red social a ser responsabilizada de acontecimientos de gran magnitud geopolítica, como la llegada de Donald Trump a la Presidencia de Estados Unidos. Es decir, su función como generador y difusor de noticias se ha ido incrementando hasta convertirse en el lugar al que millones de personas acuden a diario en busca no solo de información sino de opinión.

Consideramos que esta transformación es un motivo científico de peso para que desde la Academia se siga analizando en profundidad el fenómeno de Facebook. En este trabajo, vamos a cuestionar la versión oficial que afirma que el propietario de esta plataforma es Mark Zuckerberg. Aplicando la perspectiva crítica estructural, el objetivo de este trabajo es el análisis de la estructura de propiedad de Facebook desde el enfoque de la Economía Política de la Comunicación y la cultura (EPICC).

\subsection{Un nuevo canal mensajístico}

Facebook fue fundada por los jóvenes Mark Elliot Zuckerberg, Dustin Moskovitz, Chris R. Hughes, Andrew McCollum y Eduardo P. Saverin en la Universidad de Harvard para conectar a 
los estudiantes de la institución. A día de hoy, la website de la empresa afirma lo siguiente con respecto a su función social: "Founded in 2004, Facebook's mission is to give people the power to build community and bring the world closer together. People use Facebook to stay connected with friends and family, to discover what's going on in the world, and to share and express what matters to them" ${ }^{1 "}$.

La plataforma ha adquirido tanta relevancia social que en su informe de finales de 2016 reportó un promedio de 1,18 billones de usuarios activos ${ }^{2}$. Para esta elevada cifra de personas, Facebook ya no es solo una red para conectarse con otros usuarios individuales y grupales, pues, según el Pew Research Center, el $44 \%$ de los norteamericanos se informa principalmente a través de Facebook ${ }^{3}$. Es decir, en poco más de una década se ha transformado hasta desarrollar funciones propias de la prensa tradicional, como producir y difundir noticias. Por lo tanto, crea opinión pública, la cual puede modificar el pensamiento y la acción de determinadas personas y/o comunidades (Sartori, 2005: 176).

Debido a la asunción de estas funciones, la plataforma ha sido vinculada no sólo con la generación de información sino con el fenómeno de la desinformación y las Fake News (Allcott y Gentzkow, 2017).

Un ejemplo que muestra su protagonismo como centro generador y difusor de noticias es que un mes después de la derrota de Hillary Clinton, Zuckerberg aseguró en su perfil personal que Facebook dispondría de una nueva aplicación para advertir a los usuarios de que podrían estar leyendo o compartiendo noticias falsas ${ }^{4}$. En su discurso agregó: "Aunque nosotros no escribimos las noticias que lees y compartes, reconocemos que somos más que un distribuidor de noticias. Somos un nuevo tipo de plataforma para el debate público y eso significa que tenemos un nuevo tipo de responsabilidad a la hora de permitir que la gente mantenga las conversaciones más relevantes y para construir un espacio donde la gente pueda estar informada" ${ }^{.}$.

Es decir, una derrota electoral —la de Hillary Clinton frente a Donald Trump - provocó que Zuckerberg reconociera públicamente que Facebook no es sólo "un distribuidor de noticias" sino "un nuevo tipo de plataforma para el diálogo público". Esta afirmación dista mucho de lo que defendió sólo unos meses antes, cuando en agosto de 2016 aseguró que la empresa únicamente era una "plataforma tecnológica" y no un medio de información ${ }^{6}$.

Por su relevancia social en la vida de millones de personas en el mundo — debido a su función generativa de noticias y de opinión pública-, consideramos necesario un análisis en profundidad de Facebook desde la EPICC. Nuestro propósito es desarrollar una serie de trabajos académicos que esclarezcan la verdadera naturaleza e intencionalidad mensajística de dicha empresa, pues en toda estructura de comunicación "la correlación estructura-mensaje es evidente" (Reig, 2013). Comenzaremos, como ya hemos adelantado, por el análisis estructural de propiedad. 


\section{OBJETIVOS Y METODOLOGÍA}

A continuación vamos a exponer los objetivos que perseguimos con nuestro trabajo, así como la metodología aplicada para conseguirlo.

\subsection{Objetivos}

El objetivo de nuestro trabajo —que se inserta en la línea analítica estructural acerca de los vínculos entre la Comunicación y el Poder que venimos desarrollando- es esclarecer qué clase de estructura define realmente a Facebook Inc. Para ello, consideramos primordial analizar su estructura de propiedad. Por lo tanto, este no es un trabajo teórico sino que pretendemos aportar una aplicación práctica referente a identidad de los dueños de esta compañía.

Ya está suficientemente demostrado en la Academia que la naturaleza de la propiedad de los medios de comunicación tradicionales condiciona el proceso informativo (Schiller, 1976; Curran, 1977; Reig, 2011; Mancinas, 2009), pero en cada revolución industrial surgen nueva canales y formatos mediáticos. En este sentido, y como hemos apuntado en la introducción, Facebook ya está siendo reconocido como un centro generador de noticias y de opinión, por ello consideramos necesario analizar su naturaleza completa. Es decir, qué hay más allá de la aparente propiedad atribuida a Zuckerberg. ¿Es él su dueño o, simplemente, la cara visible de un entramado económico-financiero más complejo?

Dentro de nuestros objetivos a largo plazo se encuentra el seguir investigando y exponiendo otros aspectos estructurales de esta compañía, pero el objetivo de este trabajo concreto es analizar su estructura de propiedad.

Dentro del objetivo de este trabajo buscamos concienciar acerca de la importancia de conocer la naturaleza de los difusores de los mensajes que recibimos, debido a su injerencia en la creación de la opinión pública generada respecto a los temas que, como humanos y ciudadanos, nos afectan. Como destaca Sartori: "Cuando afirmamos que en las democracias el público se forma una opinión propia de la cosa pública, no afirmamos que el público lo haga todo por sí mismo y solo. Sabemos muy bien, por tanto, que existen 'influyentes' e 'influenciados', que los procesos de opinión van desde los primeros a los segundos, y que en el origen de las opiniones difusas están siempre pequeños núcleos de difusores" (2005: 176).

Por lo tanto, conocer a los propietarios de Facebook nos llevará a conocer a los difusores de algunos de los mensajes lanzados en su plataforma.

\subsubsection{Metodología}

La metodología y la teoría que fundamentan este trabajo tienen su base en el enfoque estructural. Lo elegimos porque este campo de trabajo proporciona las herramientas precisas para ir más allá de la apariencia, ya que se trata de la "línea de trabajo que intenta describir y explicar las 
conexiones que existen entre los grandes grupos de comunicación y otros sectores económicos y políticos, visibles o invisibles. Es decir, la vinculación entre la estructura mediática y la estructura político-económica a nivel planetario, aunque muchas veces por cuestiones metodológicas se parcelen los estudios" (Labio-Bernal, 2006:13-14).

La utilidad de nuestro enfoque metodológico consiste, sobre todo, en el hecho de "ser conscientes de lo que nos rodea" (Mancinas-Chávez, 2009:39). Por ello, consideramos que el enfoque estructural es el pertinente para que desde la Academia contribuyamos —como intelectuales comprometidos en la línea de la Escuela de Frankfurt- a que los usuarios de esta plataforma sean conscientes de quién hay detrás de la cara visible de Mark Zuckerberg.

Uno de los campos que analiza el enfoque estructural es el de la propiedad de los medios de comunicación. Conocer a los propietarios de los medios y canales que desarrollan tareas propias del periodismo es un objetivo fundamental para confirmar si el ejercicio de la comunicación está siendo interrumpido por algún condicionante que impida el proceso (Reig, 2011).

Desde los análisis de los años 50 de Mills (1993), los científicos críticos han demostrado que los propietarios de los medios de comunicación pertenecen a la elite económica y que condicionan el contenido que los medios ofrecen. Es decir, el mecanismo por el que el Poder logra controlar la producción de mensajes es convirtiéndose en el propietario directo o indirecto de los medios. De este modo, el análisis de la $\mathrm{P}$ de Propiedad de Facebook, obtenido de la Fórmula de las 6 Pes que Reig expone en Crisis del sistema, crisis del periodismo (2015), revelará la estructura económica y, por lo tanto, de poder que se esconde detrás de la parte visible de Facebook Inc.

\section{LA JUNTA DIRECTIVA DE FACEBOOK}

Vamos a comenzar nuestro trabajo investigando a la Junta Directiva de Facebook con el fin de encontrar a los miembros que la componen. Seguimos así el patrón que Reig explica en Los dueños del periodismo: "lo interesante es descubrir los nombres de los principales consejeros del grupo ${ }^{7}$ y vincular los distintos sectores industriales que convergen en el negocio de la comunicación" (2011:147). En cada uno de los sujetos de nuestra muestra vamos a comprobar cómo confluye el entramado de intereses empresariales que conforman la "telaraña mediática" (Reig, 2010).

Según la website de la compañía ${ }^{8}$, la composición de la Junta directiva de Facebook está integrada por los siguientes nombres: Mark Zuckerberg, Marc Andreessen, Susan Desmond-Hellmann, Donald E. Graham, Reed Hastings, Erskine Bowles, Peter Thiel, Sheryl Sandberg y Jan Koum. 
Tabla 1. Junta directiva de Facebook

\begin{tabular}{|l|l|}
\hline \multicolumn{2}{|c|}{ Board of Directors } \\
\hline Mark Zuckerberg & Founder, Chairman and CEO, Facebook \\
Marc Andreessen & Co-founder and General Partner, Andreessen Horowitz \\
Susan Desmond-Hellmann & CEO, Bill and Melinda Gates Foundation \\
Donald E. Graham & Chairman and CEO The Washington Post Co. \\
Reed Hastings & Chairman and CEO, Netflix \\
Erskine Bowles & President Emeritus, the University of North Carolina \\
Peter Thiel & Partner, Founders Fund \\
Sheryl Sandberg & COO, Facebook \\
Jan Koum & Founder and CEO, WhatsApp \\
\hline
\end{tabular}

Fuente: Elaboración propia a partir de datos de la web oficial de la compañía, 2017.

Comprobamos que hay varios directivos vinculados a medios de comunicación e industrias del entretenimiento en la junta. Los directamente conectados son: Donald E. Graham, Chairman and CEO de The Washington Post Co., y Reed Hastings, Chairman and CEO de Netflix. Otros pertenecen a fondos de inversión, al ámbito Académico o a fundaciones. Se cumple aquí el fenómeno de los "directorios interconectados" (Dreier y Weinberg, 1979; Brandeis, 1913).

En su web destacan, además, que el liderazgo de la compañía recae en cinco personas: Mark Zuckerberg, Sheryl Sandberg, David Wehner, Mike Schroepfer y Chris Cox.

Tabla 2. Liderazgo en Facebook

\begin{tabular}{|l|}
\hline \multicolumn{1}{|c|}{ Liderazgo en Facebook } \\
\hline Mark Zuckerberg: Fundador, presidente y consejero delegado \\
Sheryl Sandberg: Directora general \\
David Wehner: Director financiero \\
Mike Schroepfer: Director tecnológico y vicepresidente de ingeniería \\
Chris Cox: Director general de producto
\end{tabular}

Fuente: Elaboración propia a partir de los datos de Facebook.com ${ }^{9} 2017$.

El análisis de cada uno de sus miembros sería un trabajo de "directorios interconectados" lo suficientemente interesante y amplio para ser desarrollado en otro artículo. Ahora vamos a centrarnos en algunos de los nombres que ha arrojado esta primera indagación, como el de la directora general, Sheryl Sandberg. En la website de la compañía encontramos los siguientes datos: "Antes de formar parte de Facebook, Sheryl había sido vicepresidenta de ventas en internet y operaciones globales de Google, jefa de personal del Departamento del Tesoro estadounidense bajo el mandato de Bill Clinton, consultora de gestión en McKinsey \& Company y economista en el Banco Mundial. (...) También forma parte de los consejos de Facebook, Walt Disney 
Company, Women for Women International, ONE, V-Day y el Centro para el Desarrollo Global estadounidense".

Comprobamos que, en el pasado, Sandberg ha estado vinculada al Banco Mundial y a la política como jefa de personal del Departamento del Tesoro con Bill Clinton y, en la actualidad, compagina su cargo en Facebook con el de consejera en Walt Disney Company, uno de los seis grandes conglomerados mediáticos mundiales, cumpliéndose el paradigma de los "directorios interconectados" y la "telaraña mediática" (Reig, 2010).

\subsection{Peter Thiel, uno de los primeros inversores}

En 2008, el periodista de The Guardian Tom Hodgkinson publicó un reportaje de investigación sobre Facebook en el que expuso los vínculos existentes de esta empresa con la CIA y su política de espionaje global. Lo tituló "With friends like these..."10 y en él describió a los tres miembros que entonces estaban en la Junta Directiva de Facebook: Mark Zuckerberg, Peter Thiel y James Breyer, de la firma de capital riesgo Accel Partners.

Thiel es el cofundador y CEO del banco en línea PayPal y fue el primero en invertir en esta red social. Como destaca Hodgkinson en el mismo artículo: "invirtió 500.000 dólares en Facebook cuando los estudiantes de Harvard, Zuckerberg, Chris Hughes y Dustin Moskowitz se reunieron con él en San Francisco en junio de 2004, poco después de haber lanzado el sitio. Thiel posee ahora el 7\% de Facebook, que, en la valoración actual de \$15bn, valdría más de \$1 billón".

Aunque en Silicon Valley se le considera un genio libertario, "es el cofundador y CEO del sistema bancario virtual PayPal, que vendió a Ebay por $\$ 1.5 \mathrm{bn}$, quedándose $\$ 55 \mathrm{~m}$ para él. También administra un fondo de capital de 3 mil millones de libras llamado Clarium Capital Management y un fondo de capital riesgo denominado Founders Fund. La revista Bloomberg Markets lo calificó recientemente como "uno de los gestores de fondos de cobertura más exitosos del país"” (Hodgkinson, 2008).

Por ser uno de los primeros accionistas de Facebook, queremos saber si está conectado a otras estructuras de poder. Hemos buscado su nombre en las listas de asistentes a Bilderberg Club (Martín-Jiménez, 2017), comprobando su presencia en los siguientes años: 
Tabla 3. Peter Thiel en Bilderberg

\begin{tabular}{|l|}
\hline \multicolumn{1}{|c|}{ Liderazgo en Facebook } \\
\hline 2007, 2008, 2009, 2010, 2011 \\
USA Thiel, Peter A. President, Clarium Capital Management, LLC \\
2012 \\
USA Thiel, Peter A. President, Clarium Capital/ Thiel Capital \\
2013, 2014, 2015, 2016 \\
USA Thiel, Peter A. President, Thiel Capital
\end{tabular}

Fuente: Elaboración propia con datos de Martín-Jiménez (2017) y la web oficial de Bilderberg ${ }^{11}, 2017$.

Como podemos observar, Thiel estuvo en Bilderberg por primera vez en 2007, por lo que lleva más de una década formando parte de la entidad. De modo que las relaciones internacionales de uno de los miembros de la Junta Directiva de Facebook Inc. se desarrollan al más alto nivel socio-político-mediático-mercantil ${ }^{10}$.

La Junta Directiva de Facebook nos lleva a Bilderberg a través de Peter Thiel. Y también a través de uno de sus fundadores, Chris R. Hughes, que aparece en la lista oficial de la los invitados a la reunión de $2011^{13}$.

Tabla 4. Chris R. Hughes en Bilderberg

\begin{tabular}{|ll|}
\hline \multicolumn{2}{|c|}{ Liderazgo en Facebook } \\
\hline 2011 USA Hughes, Chris R. Co-founder, Facebook \\
\hline
\end{tabular}

Fuente: Elaboración propia, 2017.

Hughes fue el director de organización de las redes sociales e internet de la campaña electoral de Barack Obama en 2008, año que le llevó a la Casa Blanca. La website que creó fue BarackObama.com, que aún sigue activa.

Martín-Jiménez afirma la asistencia de Mark Zuckerberg y de su hermana Randi, directora de marketing de la compañía, en ese mismo año (2017). Pero no son los únicos directivos de Facebook vinculados a la entidad. Sin embargo, para evitar extendernos, dejaremos para un próximo trabajo las conexiones entre Bilderberg y Facebook.

\subsubsection{James Breyer, Facebook y News Corp.}

Otro de los primeros accionistas de la compañía, que ya hemos citado, es James Breyer, fundador y director ejecutivo de Breyer Capital, una firma de inversión diversificada global. Thiel era su socio en la firma de capital de riesgo Accel Partners, creada en 1987 y disuelta en 2016. Y Breyer 
también "invirtió \$12,7 millones en Facebook en abril de 2005" (Hodgkinson, 2008), en los inicios de la compañía. En 2013, después de ocho años, abandonó la Junta de Facebook ${ }^{14}$ para centrarse en otros negocios, según manifestó, y se incorporó al órgano de Gobierno de la Universidad de Harvard. Pero su curriculum aporta más datos en el campo económico-financiero: fue Director de Blackstone Group LP hasta 2016, Director de Wal-Mart Stores, Inc. de 2001 a 2013, Director de Dell, Inc. de 2009 a 2013, Director de Marvel Entertainment, Inc. de 2006 a 2009 y Director de RealNetworks, Inc. de 1995 a 200, entre otros ${ }^{15}$.

Además de sus nexos pasados con el campo del entretenimiento en Marvel, Breyer está actualmente vinculado al sector mediático a través de News Corp., pues es miembro de la Junta Directiva de 21st Century Fox, a la que entró en 2011, según la web de la compañía ${ }^{16}$.

Otros accionistas procedentes de diferentes sectores, algunos de ellos aparentemente ajenos a la comunicación y el entretenimiento, se unieron a Facebook en los primeros años. En noviembre de 2007, "Facebook anunció que doce marcas mundiales habían subido a bordo, entre ellas, Coca-Cola, Blockbuster, Verizon, Sony Pictures y Condé Nast" (Hodgkinson, 2008). Públicamente afirmaron que serían anunciantes y que abrirían muros para desarrollar las relaciones públicas con sus clientes y usuarios de Facebook.

Por su parte, Microsoft, que en 2007 compró un 1,6\% de Facebook por 240 millones de dólares ${ }^{17}$, es socio de la compañía en otros sectores.

\section{LOS ACCIONISTAS DE FACEBOOK INC.}

A continuación, vamos a exponer la estructura accionarial de Facebook, lo que nos seguirá revelando su estructura de propiedad. La fuente a la que hemos recurrido para la obtención de datos financieros ha sido el portal Yahoo Finance. Después de cotejar la información que ofrecían otros sitios, comprobamos que no sólo eran coincidentes sino que la de Yahoo Finance se mostraba mucho más completa. De modo que la citamos como referencia por considerarla la mejor de todas las que hemos consultado. Estos eran los accionistas individuales de Facebook Inc. a finales de 2016, según Yahoo Finance ${ }^{18}$.

Tabla 5. Accionistas de Facebook Inc. Accionistas personales

\begin{tabular}{|l|l|l|}
\hline \multicolumn{1}{|c|}{ Name } & \multicolumn{1}{|c|}{ Shares } & \multicolumn{1}{c|}{ Date Reported } \\
\hline KOUM JAN & $2,576,396$ & Nov 16, 2015 \\
\hline SANDBERG SHERYL & $4,007,056$ & Oct 19, 2016 \\
\hline SCHROEPFER MICHAEL TODD & 679,713 & Oct 17, 2016 \\
\hline
\end{tabular}

Fuente: Elaboración propia a partir de los datos de Yahoo Finance, 2016. 
Tabla 5. Accionistas de Facebook Inc. Accionistas personales

\begin{tabular}{|l|l|l|}
\hline \multicolumn{1}{|c|}{ Name } & \multicolumn{1}{c|}{ Shares } & \multicolumn{1}{c|}{ Date Reported } \\
\hline COX CHRISTOPHER K & 397,668 & Oct 17, 2016 \\
\hline THIEL PETER & 3,778 & May 15, 2016 \\
\hline FISCHER DAVID B. & 159,353 & Oct 17, 2016 \\
\hline ATHWAL JAS & 110,943 & Aug 19, 2016 \\
\hline HASTINGS REED & 83,075 & May 15, 2016 \\
\hline
\end{tabular}

Fuente: Elaboración propia a partir de los datos de Yahoo Finance, 2016.

Respecto a los accionistas institucionales, estos son los que presenta la web citada:

Tabla 6. Accionistas de Facebook Inc. Principales accionistas institucionales

\begin{tabular}{|c|c|c|c|c|}
\hline Holder & Shares & Date Reported & $\%$ Out & Value \\
\hline Vanguard Group, Inc. (The) & $147,041,647$ & Sep 30, 2016 & $6.28 \%$ & $18,861,032,648$ \\
\hline FMR, LLC & $142,337,495$ & Sep 30, 2016 & $6.08 \%$ & $18,257,631,053$ \\
\hline Price (T.Rowe) Associates Inc & $73,723,960$ & Sep 30, 2016 & $3.15 \%$ & $9,456,572,644$ \\
\hline State Street Corporation & $63,936,547$ & Sep 30, 2016 & $2.73 \%$ & $8,201,141,139$ \\
\hline BlackRock Institutional Trust Company, N.A. & $61,107,258$ & Sep 30, 2016 & $2.61 \%$ & $7,838,228,228$ \\
\hline Capital World Investors & $34,067,024$ & Sep 30, 2016 & $1.46 \%$ & $4,369,777,304$ \\
\hline BlackRock Fund Advisors & $30,413,666$ & Sep 30, 2016 & $1.30 \%$ & $3,901,161,059$ \\
\hline JP Morgan Chase \& Company & $27,152,146$ & Sep 30, 2016 & $1.16 \%$ & $3,482,805,876$ \\
\hline Northern Trust Corporation & $26,421,414$ & Sep 30, 2016 & $1.13 \%$ & $3,389,074,879$ \\
\hline Morgan Stanley & $25,978,335$ & Sep 30, 2016 & $1.11 \%$ & $3,332,241,134$ \\
\hline
\end{tabular}

Fuente: Elaboración propia a partir de los datos de Yahoo Finance, 2016. 
Como fondos financieros, estos son los accionistas de Facebook Inc.:

Tabla 7. Accionistas de Facebook Inc. Principales fondos

\begin{tabular}{|c|c|c|c|c|}
\hline Holder & Shares & Date Reported & $\%$ Out & Value \\
\hline Fidelity Contrafund Inc & $52,435,684$ & Aug 31, 2016 & $2.24 \%$ & $6,613,188,623$ \\
\hline $\begin{array}{l}\text { Vanguard Total Stock Market Index } \\
\text { Fund }\end{array}$ & $43,803,673$ & Jun 30, 2016 & $1.87 \%$ & $5,005,883,706$ \\
\hline Vanguard 500 Index Fund & $30,327,308$ & Jun 30, 2016 & $1.30 \%$ & $3,465,804,727$ \\
\hline $\begin{array}{l}\text { Vanguard Institutional Index Fund-Insti- } \\
\text { tutional Index Fund }\end{array}$ & $25,795,794$ & Jun 30, 2016 & $1.10 \%$ & $2,947,943,312$ \\
\hline SPDR S\&P 500 ETF Trust & $24,389,388$ & Aug 31, 2016 & $1.04 \%$ & $3,075,989,687$ \\
\hline $\begin{array}{l}\text { Powershares Exhg Traded Fd Tr-Power- } \\
\text { shares QQQ Tr, Series } 1\end{array}$ & $16,523,041$ & Aug 31, 2016 & $0.71 \%$ & $2,083,885,980$ \\
\hline Price (T.Rowe) Growth Stock Fund Inc. & $16,458,298$ & Sep 30, 2016 & $0.70 \%$ & $2,111,105,950$ \\
\hline Growth Fund Of America Inc & $15,765,000$ & Sep 30, 2016 & $0.67 \%$ & $2,022,176,613$ \\
\hline $\begin{array}{l}\text { Price (T.Rowe) Blue Chip Growth Fund } \\
\text { Inc. }\end{array}$ & $12,935,800$ & Sep 30, 2016 & $0.55 \%$ & $1,659,275,117$ \\
\hline Fidelity 500 Index Fund & $12,416,623$ & Aug 31, 2016 & $0.53 \%$ & $1,565,984,530$ \\
\hline
\end{tabular}

Fuente: Elaboración propia a partir de los datos de Yahoo Finance, 2016.

En estas tres tablas comprobamos que, en la actualidad, los propietarios de Facebook, lejos de ser un grupo de jóvenes idealistas, son fondos de capital, instituciones financieras y algunos de los hombres más ricos del mundo, como Jan Koum, CEO y fundador de WhatsApp, la aplicación de mensajería móvil que Facebook Inc. adquirió en febrero de 2014 por 19.000 millones de dólares.

\section{LOS VÍNCULOS DE FACEBOOK CON EL FONDO DE CAPITAL DE LA CIA}

En el reportaje de investigación de The Guardian anteriomente citado, el periodista Tom Hodgkinson se refirió, además, a las conexiones de Facebook con la $\mathrm{CIA}^{19} \mathrm{y}$ a sus implicaciones con el espionaje global. En 2013, uno de los trabajadores de la National Security Agency, Edward Snowden, desvelaría la existencia del programa de vigilancia masiva PRISM, en el que participaban las siguientes empresas de tecnología: Facebook, Microsoft, Yahoo, Google, PalTalk, AOL, Skype, YouTube y Apple ${ }^{20}$. 
Hodgkinson afirmó en su artículo que la última financiación recibida por Facebook procedía de "Greylock Venture Capital, con 27,5 millones de dólares" y se expresó en estos términos para exponer las vinculaciones de este fondo de capital: "Uno de los socios senior de Greylock es Howard Cox, ex presidente de la NVCA, que también está en la Junta Directiva de In-Q-Tel. ¿Qué es In-Q-Tel? Bueno, lo crean o no (y echen un vistazo a su sitio web) es el ala de capital riesgo de la CIA ${ }^{21 "}$ (Hodgkinson, 2008).

Es decir, Howard Cox es, al mismo tiempo, accionista de Facebook y de In-Q-Tel, siendo directivo de este último.

La CIA tiene su propia firma de capital riesgo para realizar inversiones: In-Q-Tel Inc. ${ }^{22}$. Según leemos en su website, In-Q-Tel fue creada en 1999 para trabajar con "empresas que desarrollan tecnologías de vanguardia para ayudarla a entregar estas soluciones a la Agencia Central de Inteligencia".

En 2009, The Telegraph ${ }^{23}$ informó que la CIA estaba invirtiendo en Visible Technologies a través de In-Q-Tel, la firma de software especialista en monitorear websites, blogs, Twitter, YouTube. Además, los espías estadounidenses estaban interesados en conocer qué libros leía la gente, por lo que también husmeaban en Amazon. Dos de los nombres que se presentan con mayor frecuencia en relación con In-Q-Tel eran Google y Facebook ${ }^{24}$.

Por otra parte, entre las acciones y revueltas políticas que se han ejercido a través de Facebook en diferentes partes del mundo, Pascual Serrano ha analizado las movilizaciones sociales contra la guerrilla de las Fuerzas Armadas Revolucionarias de Colombia (FARC) en febrero de $2008^{25}$.

Además de la NSA y la CIA, entre otras relaciones de alto nivel de Zuckerberg destaca la que mantiene con Barack Obama, con quien durante su presidencia del Gobierno ha celebrado encuentros y conferencias en Universidades de EEUU ${ }^{26}$. Ya hemos destacado quién dirigía sus redes sociales cuando ganó las elecciones.

Y ya para finalizar, y aunque dejaremos para próximos trabajos los vínculos de Facebook Inc. con otros sectores productivos, queremos dejar constancia de que es socio de Microsoft en varios negocios como MAREA ${ }^{27}$, el cable subacuático que cruzará el Atlántico desde Bilbao hasta Virginia para sustentar "la creciente demanda de conexiones inalámbricas y de alta velocidad para servicios cloud y en línea para Microsoft, Facebook y sus clientes", según manifestó el director de la compañía Frank Rey ${ }^{28}$. El proyecto fue diseñado conjuntamente por ambas empresas junto a Telxius, la compañía de infraestructura de telecomunicaciones de Telefónica.

Nuestro objetivo por dejar constancia de que estas son las compañías que instalarán dicho cable submarino se debe a los precedentes en el campo del espionaje de Facebook y Microsoft y a que debemos ser conscientes de ello. 


\section{CONCLUSIONES}

En el desarrollo de nuestro análisis hemos encontrado a entidades y personalidades procedentes del ámbito económico-financiero, del político, el espionaje y del mediático en la estructura de propiedad de Facebook. La primera conclusión a la que nos llevan estos datos es que Mark Zuckerberg no es su propietario sino, únicamente, la cara visible de la compañía. La parte de invisible de la estructura de propiedad son los fondos de inversión y el resto de accionistas citados, como la CIA. Un ejemplo paradigmático de esta invisibilidad se manifestó cuando fue Zuckerberg quien acudió al Congreso de los EEUU para explicar lo ocurrido con Cambridge Analityca ${ }^{29}$ en vez de los propietarios de los grandes fondos financieros que conforman su accionariado. $\mathrm{O}$ de la misma CIA.

Ya demostramos en nuestra tesis doctoral (Martín-Jiménez, 2017) que los grandes fondos de inversión están presentes en la estructura de propiedad de los conglomerados de comunicación globales. En este trabajo confirmamos que también lo están en la estructura de Facebook.

Una vez analizada la estructura de propiedad, entre la que se encuentra la CIA podemos obtener otras conclusiones que sostiene el enfoque de la EPICC. Ya es claro que Facebook no es sólo una red social ni tampoco únicamente una empresa global dedicada a vender publicidad. Que los grandes financieros, la CIA, la estructura política y mediática, así como miembros de Bilderberg se interesaran por convertirse, desde el inicio, en sus propietarios, unido a los hechos demostrados de que el programa PRISM lo usó para espionaje global, que Cambridge Analytica se hiciera con los datos de sus usuarios y el estudio de Pascual Serrano revelan las potencialidades y capacidades de Facebook para ejercer la manipulación social.

Consideramos que Facebook es un producto de la Cuarta Revolución Industrial que aún no ha revelado todo su potencial como manipulador de individuos y sociedades globales. No solo tiene capacidad para vender productos mercantiles a través de la publicidad, sino candidatos a la presidencia de un Gobierno o impulsar acciones de activismo político-social, como el caso de Colombia analizado por Serrano. Es también una plataforma a la que recurre la CIA para vigilar a sus usuarios.

Zuckerberg es solo la cara visible de una compañía integrada en la telaraña mediática global, donde hemos encontrado al Club Bilderberg, la CIA, la NSA, a fundaciones como la de Bill y Melinda Gates, a miembros de la elite económica global, así como de la elite financiera, política y académica, como Erskine Bowles, el President Emeritus de la University of North Carolina.

Como apuntamos al inicio, Facebook se ha convertido con el paso del tiempo en mucho más que una simple red social. Sin embargo, después de este trabajo, surgen nuevas incógnitas a resolver, como la pregunta acerca de si desde su lanzamiento, en 2004, ya contenía un mecanismo implícito para hacer ingeniería social, es decir, con el fin de manipular y controlar la acción o inacción de personas, grupos y sociedades. En este sentido, es evidente que la imposibilidad de acceder a esta información nos impide realizar afirmaciones categóricas a este respecto. Pero queremos 
manifestar las nuevas preguntas científicas que nos surgen después de haber descubierto su estructura de propiedad y la necesidad de seguir investigando a esta plataforma.

Tras lo expuesto, otras conclusiones obtenidas son las siguientes:

- Al igual que los grandes conglomerados, corporaciones y grupos de la comunicación, Facebook mantiene múltiples conexiones con la banca, el sector militar, las nuevas tecnologías, la Academia y los sectores productivos (a través de la publicidad), entre otros. Hemos hallado, por lo tanto, una confluencia de intereses en la estructura de propiedad de Facebook.

- En Facebook encontramos connivencia entre el poder político, el militar, el económico y el informativo.

- En Facebook se cumplen las características de los conglomerados mediáticos globales: la diversificación del capital, la concentración del poder económico y la globalización. Si la correlación entre intereses de propiedad, orden socioeconómico mundial y mensajes periodísticos es evidente (Reig, 2011), hay que analizar los mensajes que lanzan los propietarios de Facebook en esta plataforma y en otros medios de comunicación.

- La opacidad que caracteriza a los fondos de inversión de capital impide llegar hasta los nombres de todos sus accionistas.

Sin embargo, en esta coyuntura sí es importante subrayar que hemos logrado acceder a la estructura de propiedad de Facebook comprobando que ésta es eminentemente de carácter mercantil. Por lo tanto, sí hemos conseguido esclarecer en qué ámbito y a qué sinergias puede obedecer una plataforma que se presenta con una cara amable y democrática, pero cuyos propietarios son algunos de los conglomerados financieros más poderosos del mundo, como por ejemplo The Vanguard Group, Inc. o Price (T. Rowe) Associates Inc.

Por todo lo expuesto, queda claro que se requieren más estudios académicos en profundidad de dicha compañía, pues detrás de una red social con apariencia de democratización existe una estructura de poder financiero. El poder siempre persigue fines concretos y estos, según han demostrado los críticos del enfoque estructural, consisten esencialmente en permanecer en el poder.

Detrás de toda plataforma de comunicación y de sus noticias encontramos los intereses de sus propietarios. Facebook es una plataforma, un nuevo canal de Comunicación de carácter privado no público. Como todo negocio, sus propietarios tienen un interés mercantil. Sin embargo, al ser un negocio del ámbito de la Comunicación, tienen, además, una intencionalidad. Los innumerables trabajos presentados por los científicos críticos de la EPICC han demostrado que el objetivo de los propietarios de los grandes medios es influir en la opinión pública para seguir manteniéndose en el poder. Facebook es un producto de las elites económicas y estas mantienen lazos de connivencia con las elites de todos los sectores a través de sus "directorios interconectados", con el fin de perpetuarse inalterable como estructura de poder. 


\section{NOTAS}

1 "Fundada en 2004, la misión de Facebook es dar a las personas el poder de construir una comunidad y unir al mundo. La gente usa Facebook para mantenerse en contacto con amigos y familiares, para descubrir qué está pasando en el mundo y para compartir y expresar lo que les importa". Consultado el 04/05/2017.

2 https://investor.fb.com/investor-news/press-release-details/2016/Facebook-Reports-third-Quarter-2016Results/default.aspx Consultado el 05/06/2017.

${ }^{3} \mathrm{http}: / /$ www.lavanguardia.com/internacional/20161115/411844638270/facebook-victoria-donald-trump. html Consultado el 20/11/2016.

${ }^{4}$ https://www.facebook.com/zuck/posts/10103338789106661 Consultado el 04/01/2017.

${ }^{5}$ Zuckerberg agregó que las noticias señaladas por la compañía como falsas no podrán ser promocionadas por Facebook.

${ }^{6}$ Op. Cit.

7 Aunque en ese trabajo se estaba refiriendo a Televisa, la escuela de Reig lo hace extensible a todos los grupos, corporaciones y conglomerados de comunicación existentes.

8 http://newsroom.fb.com/company-info/ Consultado el 21/12/2017.

${ }^{9}$ http://es.newsroom.fb.com/company-info/ Consultado el 16/12/2016

10 https://www.theguardian.com/technology/2008/jan/14/facebook Consultado el 10/11/2017

11 www.bilderbergmeeting.org

12 En septiembre de 2016 Facebook anunció que los usuarios ya podían realizar compras a través de la aplicación de mensajería instantánea de Facebook, Messenger, y pagar a través de Paypal o Stripe. Fuente: http://www.channelbiz.es/2016/09/13/facebook-anade-un-boton-de-comprar-a-su-messenger/ Consultado el 20/12/2016

13 Op. Cit.

14 http://allthingsd.com/20130426/jim-breyer-to-leave-facebook-board-in-june/ Consultado el 20/12/2016

15 https://www.21cf.com/management/board-of-directors/james-w-breyer/

${ }^{16}$ https://www.21cf.com/managment/james-w-breyer Consultado el 20/12/2016

17 http://www.businessinsider.com/facebook-and-microsoft-seem-to-be-parting-ways-2014-12 Consultado el $21 / 12 / 2016$

18 https://finance.yahoo.com/quote/FB/holders?p=FB Consultado el 21/12/2016

19 https://www.theguardian.com/technology/2008/jan/14/facebook Consultado el 20/12/2016 
20 https://www.washingtonpost.com/investigations/us-intelligence-mining-data-from-nine-us-internetcompanies-in-broad-secret-program/2013/06/06/3a0c0da8-cebf-11e2-8845-d970ccb04497_story. html?utm_term=.c230c946321c Consultado el 20/12/2016

21 https://www.theguardian.com/technology/2008/jan/14/facebook Consultado el 1/11/2016.

22 Paletta, Damian (2016), “The CIA's Venture-Capital Firm, Like Its Sponsor, Operates in the Shadows”, http:// www.wsj.com/articles/the-cias-venture-capital-firm-like-its-sponsor-operates-in-the-shadows-1472587352 publicado el 30/08/2016 Consultado el 19/12/2016

23 http://www.telegraph.co.uk/news/worldnews/northamerica/usa/6389669/US-spies-invest-in-internetmonitoring-technology.html Consultado el 11/12/2016

24 https://www.corbettreport.com/meet-in-q-tel-the-cias-venture-capital-firm-preview/ Consultado el $11 / 12 / 2016$

25 http://pascualserrano.net/es/noticias/bfquien-esta-detras-de-facebook-el-portal-desde-el-que-surgio-lamovilizacion-internacional-contra-las-farc Consultado el 11/12/2016

26 http://elcomercio.pe/mundo/eeuu/facebook-presidente-obama-entrevistara-mark-zuckerberg-esteviernes-noticia-1911747 Consultado el 1/12/2016

27 https://blogs.technet.microsoft.com/hybridcloud/2016/05/26/microsoft-and-facebook-to-build-subseacable-across-atlantic/ Consultado el 13/01/2016

28 https://blogs.technet.microsoft.com/hybridcloud/2016/05/26/microsoft-and-facebook-to-build-subseacable-across-atlantic/ Consultado el 13/01/2016

29 https://elpais.com/internacional/2018/04/11/actualidad/1523452080_125289.html Consultado el $12 / 04 / 2018$

\section{REFERENCIAS}

Allcott, H. and Gentzkow, M. (2017). "Social Media and Fake News in the 2016 Election". Journal of Economic Perspectives, Vol.31, Number 2, Spring 2017, Pages 211-236.

Brandeis, L. (1913): "Breaking the Money Trust". Harper's Weekly. Primera parte: "Our Financial Oligarchy" (22 noviembre, pp. 10-13). Segunda Parte: "How the Combiners Combine" (29 noviembre, pp. 9-12). Tercera parte: "The Endless Chain" (6 diciembre, pp. 13-15). Cuarta Parte: "Serve One Master Only!" (13 diciembre, pp. 10-12). Quinta parte: "What Publicity Can Do" (20 diciembre, pp. 10-13). Sexta parte: "Where the Banker is Superfluous" (27 diciembre, pp. 18-21).

Dreier, P. y Weinberg, S. (1979): “Interlocking Directorates”. Columbia Journalism Review, vol. 18, noviembre/diciembre.

Dreier, P. (1982), "The Position of the Press in the U.S. Power Structure". Social Problems, Vol. 29, n 3, febrero.

Curran, J. et al. (1977), Mass communication and society, London: The Open Universiyy Press. 
Labio-Bernal, A. (2006). Comunicación, periodismo y control informativo: Estados Unidos, Europa y España. Barcelona: Anthropos.

Mancinas-Chávez, R. (2009). La estructura mediática de México y el caso del estado de Chihuahua: prensa, radio, televisión e internet. Universidad de Sevilla.

Martin-Jiménez, C. (2017). Interrelación entre el poder socio-político-mercantil y el poder mediático mercantil: "El Club Bilderberg" (1954-2016). Universidad de Sevilla.

Mills, C. W. (1993). La elite del poder. México: Fondo de Cultura Económica.

Reig, R. (2015). Crisis del sistema, crisis del periodismo. Contexto estructural y deseos de cambio. Barcelona: Gedisa.

- (2013). "La correlación estructura socio-económico-mediática y mensajes: aportaciones desde el análisis de la comunicación mercantil”. Question, Vol. 1, n 40. Consultado en línea el 26/12/2017. http://sedici.unlp.edu.ar/bitstream/handle/10915/32846/Documento_completo. pdf?sequence $=1$

- (2011). Los dueños del periodismo. Claves de la estructura mediática mundial y de España. Barcelona: Gedisa.

- (2010). La Telaraña Mediática. Sevilla/Zamora: Comunicación Social Ediciones y Publicaciones.

Sartori, Giovanni (2005). Elementos de Teoría Política. Madrid: Alianza Editorial.

Schiller, H.I. (1976). Communications and cultural domination. White Plains. New York: International Art and Science Press.

Tejedor-Calvo, S. (2010). "Web 2.0 en los ciberdiarios de América Latina, España y Portugal”. El profesional de la información. Noviembre-diciembre 2010, vol. 19, núm. 6. 